\title{
COMPOSIÇÃO QUÍMICA, PROPRIEDADES MECÂNICAS E TÉRMICAS DA FIBRA DE FRUTOS DE CULTIVARES DE COCO VERDE ${ }^{1}$
}

\author{
ELISÂNGELA CORRADINI ${ }^{2}$, MORSYLEIDE DE FREITAS ROSA ${ }^{3}$, \\ BRUNO PEREIRA DE MACEDO ${ }^{4}$, PRISCILA DANIELLE PALADIN ${ }^{5}$, \\ LUIZ HENRIQUE CAPPARELLI MATTOSO ${ }^{6}$
}

RESUMO-O consumo da água de coco verde, in natura ou industrializada, vem gerando um grande problema ambiental, devido ao destino final da casca dos frutos. Aproximadamente $85 \%$ do peso bruto do coco verde é constituído pelas cascas, que são acumuladas em lixões ou às margens de estradas. Como a minimização da geração desse resíduo implicaria a redução da atividade produtiva associada, o seu aproveitamento tornase uma necessidade. Neste sentido, este estudo teve como objetivo investigar as características da fibra de coco verde de diversos cultivares em função do ponto de colheita dos frutos, na composição química, nas propriedades mecânicas e térmicas, como forma de contribuir para avaliar seu potencial de aplicação na elaboração de novos materiais. Os resultados mostraram que a variação da composição química em função da cultivar de coco verde foi na faixa de $37,2 \pm 0,8 \%$ a $43,9 \pm 0,7 \%$ e de $31,5 \pm 0,1 \%$ a $37,4 \pm 0,5 \%$ para os teores de lignina e celulose, respectivamente. A composição química não variou significativamente em função do ponto de maturação para a fibra da cultivar Anão-Verde-de-Jiqui (AVeJ). A fibra de cultivares de coco verde e AVeJ em diferentes pontos de maturação apresentaram propriedades térmicas e mecânicas semelhantes, as quais são próximas das propriedades das fibras de coco maduro, demonstrando, portanto, um potencial equivalente para serem utilizadas como reforço em matrizes poliméricas.

Termos para indexação: Cocos nucifera, caracterização de frutos, ponto de maturação, fibras de coco verde.

\section{CHEMICAL COMPOSITION, THERMAL AND MECHANICAL PROPERTIES FOR CULTIVARS OF IMMATURE COCONUT FIBERS}

\begin{abstract}
The consumption of water of green coconut in natura or industrialized has generated a large amount of residues that are disposed in landfills or in roadside. The husks residues correspond to around $85 \%$ of the weight of the fruit and present slow decomposition process. This environmental problem requires alternative solutions. In order to assessing to potential of the immature (green) coconut fibers to be used as reinforcement material in polymer matrices, the chemical composition, thermal and mechanical characteristics of the coconut fibers originated from 5 immature coconut cultivars of the Nana variety at 4 different ages were studied. According to the results obtained, the change of the chemical composition with the cultivar was from $37.2 \pm 0.8 \%$ to $43.9 \pm 0.7 \%$ and from $7.4 \pm 0.5 \%$ to $31.5 \pm 0.1 \%$ for lignin and cellulose contents, respectively. The age did not significantly influence the chemical composition of 'Anão Verde de Jequi' (AVeJ) cultivar. All the cultivars and AVeJ, in different ages showed approximately the same thermal and mechanical behaviors, which were similar to the mature coconut fiber properties. This study shows that the immature cultivars studied offer potential to be used as reinforcement in composites similarly to mature coconut fiber.
\end{abstract}

Index terms: Cocos nucifera, fruit characterization, immature coconut fiber, maturation point.

\footnotetext{
${ }^{1}$ (Trabalho 192-08). Recebido em: 22-07-2008. Aceito para publicação em: 03-03-2009.

${ }^{2}$ Química, Dra, Bolsista de Pós-doutorado, Embrapa Instrumentação Agropecuária, Rua XV de Novembro, 1452, CEP 13560-970, São Carlos-SP, e-mail: elisangela@cnpdia.embrapa.br

${ }^{3}$ Eng. Química, Dra, Pesquisadora, Embrapa Agroindústria Tropical, Rua Dra Sara Mesquita, 2270, CEP 60511-110, Fortaleza-CE, e-mail: morsy@cnpat.embrapa.br

${ }^{4}$ Graduando em Engenharia Química, Universidade Federal de São Carlos, Rodovia Washington Luís, km 235-SP-310, CEP 13565-905, São Carlos-SP, e-mail: brunopmacedo@yahoo.com.br

${ }^{5}$ Graduanda em Química, Universidade Federal de São Carlos, Rodovia Washington Luís, km 235 - SP-310, CEP 13565-905, São Carlos-SP, e-mail: pri paladin@hotmail.com

${ }^{6}$ Eng. Materiais, Dr, Pesquisador, Embrapa Instrumentação Agropecuária, Rua XV de Novembro, 1452, CEP 13560-970, São CarlosSP, e-mail:mattoso@cnpdia.embrapa.br. "Autor para correspondência.
} 


\section{INTRODUÇ̃̃̃O}

O consumo da água de coco e da polpa de coco gera uma quantidade significativa de resíduos, representados por suas cascas. No caso do coco maduro, as cascas são, geralmente, utilizadas como combustível de caldeiras ou ainda processadas para beneficiamento de fibras, que são usadas como manufaturas de cordoalhas, tapetes, esteiras e outros produtos (Rosa et al., 2002).

No caso do fruto imaturo ou coco verde para consumo de água, 80 a $85 \%$ do peso bruto representam resíduo (cascas), que não vêm sendo aproveitadas pela indústria de beneficiamento de fibras, devido à falta de conhecimento de suas propriedades. As cascas do coco verde têm sido descartadas nos aterros e vazadouros a céu aberto (lixões) sendo, como toda matéria orgânica, potenciais emissores de gases e, ainda, contribuindo para que a vida útil desses depósitos seja diminuída, proliferando focos de vetores transmissores de doenças, mau cheiro, possíveis contaminação do solo e corpos d'água, além da inevitável destruição da paisagem urbana (Rosa et al., 2001).

A casca do coco verde, assim como a do coco maduro, é constituída por uma fração de fibras e outra denominada de pó. As fibras de coco são materiais lignocelulósicos obtidos do mesocarpo de cocos e caracterizam-se pela sua dureza e durabilidade atribuída ao alto teor de lignina, quando comparadas com outras fibras naturais (Silva, 2006). Esse material apresenta baixa taxa de degradação, levando mais de 8 anos para completa decomposição (Carrijo et al., 2002). Como a minimização da geração desse resíduo implicaria a redução da atividade produtiva associada, o seu aproveitamento torna-se uma necessidade. No entanto, poucos estudos têm sido realizados visando à caracterização e utilização da fibra e do pó da casca do coco verde.

Um estudo realizado pela Embrapa Agroindústria Tropical mostrou viabilidade da utilização do pó da casca do coco verde como substrato agrícola (Rosa et al., 2002).

$\mathrm{Na}$ literatura, são encontrados vários estudos que foram conduzidos visando à incorporação de fibras de coco em novos materiais compósitos. Ressalta-se, entretanto, a grande predominância dos estudos voltados para uso de fibras de coco maduro (Rout et al., 2001a; Rout et al., 2001b; Silva et al., 2006; Rozman et al., 2000).

O conhecimento das características químicas, mecânicas e térmicas das fibras provenientes de frutos imaturos (coco verde) torna-se necessário para a compreensão de sua potencialidade de uso.

Este trabalho teve como objetivo investigar as características da fibra de coco verde de diversas cultivares em função do ponto de maturação dos frutos, na composição química, nas propriedades mecânicas e térmicas da fibra de coco de frutos imaturos, como forma de contribuir para avaliar seu potencial.

\section{MATERIAL E MÉTODOS}

Frutos provenientes de cinco cultivares de coqueiro (Cocos nucifera): Nana 'Anão- Verde'de-Jequi (AVeJ), 'Anão-Amarelo'-de-Gramane (AAG), 'Anão-Amarelo'-da-Malásia (AAM), 'Anão-vermelho'-da-Malásia (AVM) e 'AnãoVermelho'-de-Camarões (AVC), provenientes do Campo Experimental da Embrapa Meio-Norte, em Parnaíba-PI, Brasil, foram coletados ainda imaturos com 210 dias após a abertura natural da inflorescência. Amostras da cultivar AVeJ foram coletadas também em quatro pontos de maturação $(120 ; 150$; 180 e 210 dias). O processamento dos frutos deu-se na Embrapa Agroindústria Tropical, Fortaleza-CE, Brasil. Inicialmente, as cascas foram dilaceradas em triturador com faca de corte e martelos desintegradores; em seguida, prensadas, em prensa " $\mathrm{PRH}$ " (prensa de rolos horizontais), e classificadas, de forma a obter duas frações: pó e fibra.

Caracterização química: As fibras foram moídas em moinho de faca e passadas em peneira de 16 mesh. Foram submetidas à extração com solventes ciclohexano/etanol 1:1, por 13 horas, para remover os extrativos solúveis em solventes orgânicos e, em seguida, foram submetidas à extração com água, por 38 horas, para a remoção dos extrativos solúveis em água. Posteriormente, foram secas em estufa a $105^{\circ} \mathrm{C}$, por 6 horas, para a determinação do teor de lignina e celulose.

O teor de lignina na fibra de coco verde das cultivares foi determinado pela reação com ácido sulfúrico a $72 \%$, de acordo com a norma TAPPI T 222 om-88 (Tappi..., 1999). O teor de celulose foi determinado a partir da obtenção da holocelulose (celulose + hemicelulose) das fibras de coco verde, de acordo com o método descrito por Razera (2006), e seguida da extração da hemicelulose com solução de hidróxido de sódio, de acordo com Browing (1967). Todas as determinações foram conduzidas com três repetições e representadas pela média.

Caracterização mecânica: Os ensaios de tração para as fibras foram realizados em máquina de ensaio Instron, modelo 5569. Utilizou-se a norma ASTM D 3379-89 (American..., 1989) e as seguintes 
condições para os ensaios das fibras: velocidade de deslocamento das garras igual a $2,0 \mathrm{~mm} / \mathrm{mim}$, célula de carga de $50 \mathrm{~N}$, comprimento da fibra de aproximadamente $5,4 \mathrm{~cm}$. Foram testadas pelo menos 20 fibras para cada ensaio.

Caracterização termogravimétrica: A estabilidade térmica das fibras foi investigada por análise termogravimétrica, utilizando um aparelho TGA 500 (TA Instrument). As análises foram realizadas sob fluxo de ar sintético, com taxa de fluxo do gás de $10 \mathrm{~mL} /$ min, com taxa de aquecimento de $10^{\circ} \mathrm{C} / \mathrm{min}$, sendo a faixa da temperatura varrida de $25^{\circ} \mathrm{C}$ a $800^{\circ} \mathrm{C}$.

Microscopia Eletrônica de Varredura (MEV): A caracterização por MEV das fibras de coco verde foi realizada num Digital Scanning Microscope DSM 960 da Zeiss. As fibras foram recobertas com uma fina camada de prata e depois metalizadas com ouro num "Sputter Coater" (plasma de argônio) da Balzers modelo SCD 50.

\section{RESULTADOS E DISCUSSÃO}

\section{Composição Química}

Nas Figuras 1 e 2, são apresentados os teores de celulose, lignina e componentes secundários (hemicelulose, extrativos e cinzas) para a fibra de coco verde das cultivares AVeJ, AAM, AVC, AVM, AVG e para a cultivar AVeJ em diferentes pontos de maturação $(120 ; 150 ; 180$ e 210 dias $)$, respectivamente.

Nota-se que entre as cultivares de fibras examinadas, a composição química apresentou pequenas variações. A cultivar AVeJ foi a que demonstrou o menor teor de lignina $(37,2 \pm 0,8 \%)$, enquanto a cultivar AVG foi a que mostrou o maior teor de lignina $(43,9 \pm 0,7 \%)$. Em relação ao teor de celulose nas cultivares, verificou-se que a cultivar AAM apresentou o maior teor de celulose $(37,4 \pm 0,5 \%)$, enquanto a cultivar que apresentou o menor teor de celulose foi a AVC $(31,5 \pm 0,1 \%)$. Em termos de celulose, as fibras de coco verde apresentaram valores um pouco inferiores, comparando-se com os valores encontrados na literatura para a fibra de coco maduro (36-43\%) (Mohanty et al., 2000; Bledzki et al., 1996), e em relação ao teor de lignina, os valores obtidos são próximos aos valores observados para o coco maduro (41-45\%) (Mohanty et al., 2000). As fibras de coco verde apresentaram menores teores de celulose e maiores teores de lignina em comparação com outras fibras lignocelulósicas, tais como fibra de juta e sisal (Mohanty et al., 2000). Verifica-se que, em relação aos componentes secundários (hemicelulose, extrativos e cinzas), as fibras AVeJ, AAM, AVC e AVG apresentaram teores próximos, enquanto a fibra
AVM apresentou teor um pouco menor.

Observa-se que, para as fibras de coco verde da cultivar AVeJ, os teores de lignina mantiveramse praticamente constantes entre o período de 120 a 210 dias, e os teores de celulose apresentaram uma pequena diminuição entre o período de 120 a 150 dias, sendo que, após 150 dias, praticamente não variaram. De acordo com Trugilho et al. (1996), os teores de lignina e celulose em plantas jovens estão sujeitos a grandes variações, tendendo a valores estáveis na idade adulta. No caso em estudo, provavelmente as fibras de coco verde já atingiram valores estáveis entre o período de 150 até 210 dias. Em relação aos teores dos componentes secundários, foi observado aumento entre 120 e 150 dias de maturação, e após este período não ocorreram variações significativas.

\section{Propriedades mecânicas}

Nas Figuras 3 (a,b,c, e d) e 4 (a, b e c), são apresentados os valores de tensão na ruptura $(\sigma r)$, módulo elástico $(E)$ e deformação na ruptura $(\varepsilon)$ para as cultivares de coco AVeJ, AAM, AVC, AVM e AVG, aos 210 dias, e para a cultivar AVeJ, em diferentes pontos de maturação.

Observam-se altos valores de desvio-padrão, provavelmente atribuído à grande heterogeneidade das fibras. Entre as fibras de coco verde das cultivares, o módulo elástico (E) variou de $801 \pm 308 \mathrm{MPa}$ a $1.600 \pm 508 \mathrm{MPa}$; $\sigma$ r variou de $82 \pm 18 \mathrm{MPa}$ a 129 $\pm 40 \mathrm{MPa}$ e $\varepsilon$ de $25 \pm 6 \%$ a $32 \pm 7 \%$. Considerando a margem do erro experimental, essas variações nos valores de $\mathrm{E}$, or e $\varepsilon$ não foram significativas entre as cultivares estudadas.

Os valores de or e E são próximos aos valores mencionados na literatura para coco maduro (Mohanty et al., 2000), os quais são: $4 \times 10^{3}-6 \times 10^{3}$ $\mathrm{MPa}, 131-175 \mathrm{MPa}$, e $15-40 \%$, respectivamente, para $\mathrm{E}, \sigma \mathrm{r}, \mathrm{e} \varepsilon$. Foi verificado também que os valores de $\sigma r$ e E obtidos para fibras de coco verde são significativamente menores que os descritos na literatura para outras fibras, como juta e sisal. Isto é atribuído ao menor teor de celulose das fibras de coco (Mohanty et al., 2002). No entanto, as fibras de coco verde apresentam propriedades mecânicas superiores a vários polímeros biodegradáveis, tais como: amido termoplástico $(\mathrm{E}=70 \pm 10 \mathrm{MPa}$, or $=4,1 \pm 0,3 \mathrm{MPa}$ ) (Corradini et al., 2006), policaprolactona $(\mathrm{E}=180 \pm 15 \mathrm{MPa}, \sigma \mathrm{r}=24 \pm 5 \mathrm{MPa})$ (Corradini et al., 2004) e Ecoflex ${ }^{\circledR} \mathrm{FBX} 7011(\mathrm{E}=300 \mathrm{MPa}$, $\sigma r=13 \mathrm{MPa}$ ) (ECOFLEX, 2004). Esses resultados de $\mathrm{E}$ e $\sigma \mathrm{r}$ indicam que as propriedades mecânicas das fibras de coco verde apresentam resistência mecânica próximas das fibras de coco maduro, demonstrando 
que essas fibras possuem potencial semelhante para algumas aplicações, tais como reforço em compósitos com polímeros naturais e sintéticos.

Fibras com alto teor de lignina, como é o caso das fibras de coco, são de excelente qualidade e flexíveis. Estudos com blendas contendo lignina ou derivados têm mostrado que, em alguns casos, as propriedades mecânicas e/ou térmicas do material polimérico são melhorados com sua adição (van Dam et al., 2004).

Comparando os valores de E, or e $\varepsilon$ obtidos para a fibra de coco verde (cultivar AVeJ) em diferentes tempos de maturação, observa-se pequena variação não significativa das propriedades mecânicas em função do ponto de maturação. Os valores médios para a tensão na ruptura variaram de $116 \pm 31 \%$ a $140 \pm 18 \%$, enquanto os valores de módulo elástico variaram entre $990 \pm 262 \mathrm{MPa}$ até $1.197 \pm 301 \mathrm{MPa}$, e os valores de deformação na ruptura, de $28 \pm 5 \%$ até $35 \pm 4 \%$.

\section{Propriedades térmicas}

Nas Figuras 5a e 5b, são apresentadas curvas termogravimétricas (TG) e termogravimétricas derivadas (DTG) para as cultivares de fibra de coco verde aos 210 dias de maturação. Foi observada semelhança bastante acentuada entre as curvas TG e DTG das fibras das cultivares e também entre as fibras da cultivar AVeJ em diferentes pontos de maturação.

As curvas TG/DTG exibem uma etapa de perda de massa em torno de $25-120^{\circ} \mathrm{C}$ relativa à quantidade de água absorvida pelas amostras. A degradação térmica dos componentes das fibras de coco ocorre em uma faixa de temperatura entre 200 a $550^{\circ} \mathrm{C}$, a qual pode variar, dependendo da cultivar e das condições empregadas nas análises (Silva et al., 2000; Tomczak et al., 2007).

A hemicelulose, devido a sua natureza amorfa, decompõe-se entre $200-260^{\circ} \mathrm{C}$, enquanto a celulose, entre $240-360^{\circ} \mathrm{C}$, e a lignina, entre 280 a $360^{\circ} \mathrm{C}$ (Alvarez \& Vázquez, 2004; Yao et al., 2008). A curva DTG exibe um ombro em torno de $270^{\circ} \mathrm{C}$, que é relativo à degradação da hemicelulose, e também um pico bem definido em torno de $330^{\circ} \mathrm{C}$, devido principalmente à degradação térmica da celulose. Dois picos na faixa de 350 a $550^{\circ} \mathrm{C}$ são observados, os quais são provavelmente devido à degradação térmica da celulose e da lignina. Não é possível separar os processos de degradação dos componentes das fibras (lignina, celulose e hemicelulose) devido à complexidade das reações e da superposição dos picos de degradação.

A partir das curvas TG e DTG, foram determinados alguns parâmetros para todas as fibras estudadas: quantidade de água absorvida na faixa de $25-120^{\circ} \mathrm{C}, \mathrm{T}_{\text {onset }}$ (temperatura de início de degradação térmica) e faixa de temperatura e perda de massa (\%) relativa à degradação térmica e a quantidade de cinzas a $650^{\circ} \mathrm{C}$. Esses dados são apresentados na Tabela 1. Pode ser visto que a perda de massa no intervalo de 25 a $120^{\circ} \mathrm{C}$ variou de 4,1 a $5,8 \%$. $\mathrm{T}_{\text {onset }}$ para todas as fibras estudadas está em torno de $185-200^{\circ} \mathrm{C}$. Comparando os valores de $\mathrm{T}_{\text {onset }}$ observados para as fibras de coco verde com os valores observados para a fibra de coco maduro (Silva et al., 2000), observase que a temperatura de início de degradação foi um pouco maior para fibra de coco maduro $\left(220^{\circ} \mathrm{C}\right)$. Essa diferença pode ser devido à presença de alguns componentes voláteis nas fibras de coco verde (van Dam et al., 2004). A porcentagem de perda de massa relativa ao processo de degradação praticamente não variou para todas a fibras estudadas.

Os resultados observados por TG estão coerentes com os dados de composição química e com as propriedades mecânicas, pois, como a composição química praticamente não variou em função da cultivar e também em função do ponto de maturação para a fibra AVeJ, era esperado que a estabilidade térmica também não fosse alterada significativamente em função do tipo de cultivar e do ponto de maturação.

\section{Microscopia Eletrônica de Varredura} $(M E V)$

Nas Figuras 6a e 6b, são apresentadas fotos de microscopia eletrônica de varredura (MEV) para a fibra de coco AVeJ no ponto de maturação de 150 dias. Observa-se uma superfície rugosa, com uma camada de cera, ácidos graxos e seus produtos de condensação depositados sobre a superfície da fibra, similar à fibra de coco maduro (Bismarck et al., 2001). A rugosidade observada ao longo da superfície da fibra pode agir como regiões de ancoramento, evitando a ruptura total na interface entre a fibra e a matriz, contribuindo assim para aumentar a adesão mecânica entre a fibra e a matriz. Foi observado também que todas as fibras estudadas apresentaram aspectos morfológicos semelhantes. 
TABELA 1 -Parâmetros determinados a partir das curvas termogravimétricas (TG) e termogravimétricas derivadas (DTG) para todas as fibras estudadas: quantidade de água absorvida na faixa de 25$120^{\circ} \mathrm{C}$, Tonset (temperatura de início de degradação térmica) e faixa de temperatura e perda de massa (\%) relativa às duas etapas principais de degradação e o teor de cinzas a $650^{\circ} \mathrm{C}$.

\begin{tabular}{|c|c|c|c|c|c|c|}
\hline \multirow[b]{2}{*}{ Fibras } & \multirow[b]{2}{*}{$\begin{array}{c}\text { Ponto } \\
\text { de maturação }\end{array}$} & \multirow{2}{*}{$\begin{array}{c}\text { Quantidade de água } \\
\text { absorvida }\left(25-120^{\circ} \mathrm{C}\right) \\
\% \text { (em peso })\end{array}$} & \multirow[b]{2}{*}{$\begin{array}{l}\mathrm{T}_{\text {onset }} \\
\left({ }^{\circ} \mathrm{C}\right)\end{array}$} & \multicolumn{2}{|c|}{ Degradação térmica } & \multirow{2}{*}{$\begin{array}{c}\text { Cinzas } \\
\left(650^{\circ} \mathrm{C}\right) \\
(\%)\end{array}$} \\
\hline & & & & $\begin{array}{l}\mathrm{T}_{\mathrm{i}}-\mathrm{T}_{\mathrm{f}} \\
\left({ }^{\circ} \mathrm{C}\right)\end{array}$ & $\begin{array}{l}\text { Perda de massa } \\
\%(\text { em peso })\end{array}$ & \\
\hline AAG & 210 & 5.6 & 196 & $196-500$ & 92.64 & 2.2 \\
\hline AAM & 210 & 4.7 & 200 & $195-500$ & 92.84 & 2.0 \\
\hline AVC & 210 & 4.1 & 190 & $190-500$ & 91.88 & 4.2 \\
\hline AVM & 210 & 5.2 & 185 & $185-500$ & 91.53 & 3.0 \\
\hline AVG & 210 & 5.7 & 185 & $190-500$ & 90.98 & 3.1 \\
\hline AVeJ & 120 & 4.9 & $\begin{array}{l}100 \\
197\end{array}$ & $195-500$ & 93.62 & 1.7 \\
\hline AVeJ & 150 & 5.5 & 195 & $195-500$ & 92.57 & 2.1 \\
\hline AVeJ & 180 & 5.3 & 190 & $190-500$ & 91.67 & 2.7 \\
\hline AVeJ & 210 & 5.8 & 187 & $187-500$ & 91.60 & 2.8 \\
\hline
\end{tabular}

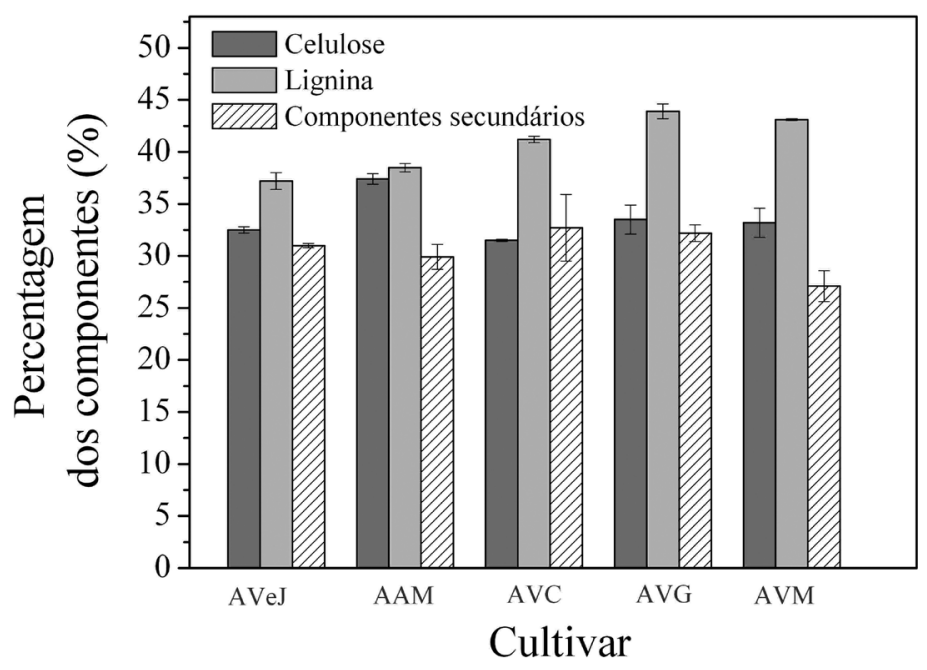

FIGURA 1 - Teor de lignina, celulose e outros componentes (hemicelulose, extrativos e cinzas) para fibras de coco verde das cultivares: AVeJ, AAM, AVC, AVG e AVM, no ponto de maturação de 210 dias. Sendo AVeJ = Nana 'Anão-Verde'-de-Jequi; AAG = 'Anão-Amarelo'-de-Gramane; $\mathrm{AAM}=$ 'Anão-Amarelo'-da-Malásia; AVM = 'Anão-Vermelho'-da-Malásia e AVC = 'AnãoVermelho'-de-Camarões.

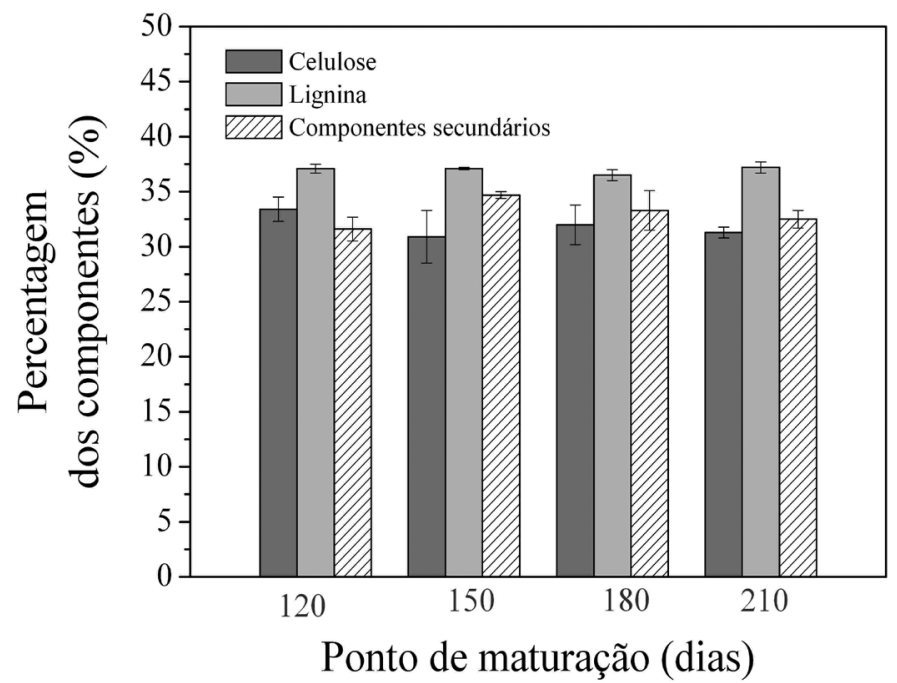

FIGURA 2 - Teor de celulose, lignina e outros componentes (hemicelulose, extrativos e cinzas) em função do ponto de maturação para a fibra Nana 'Anão-Verde'-de -Jequi (AVeJ). 

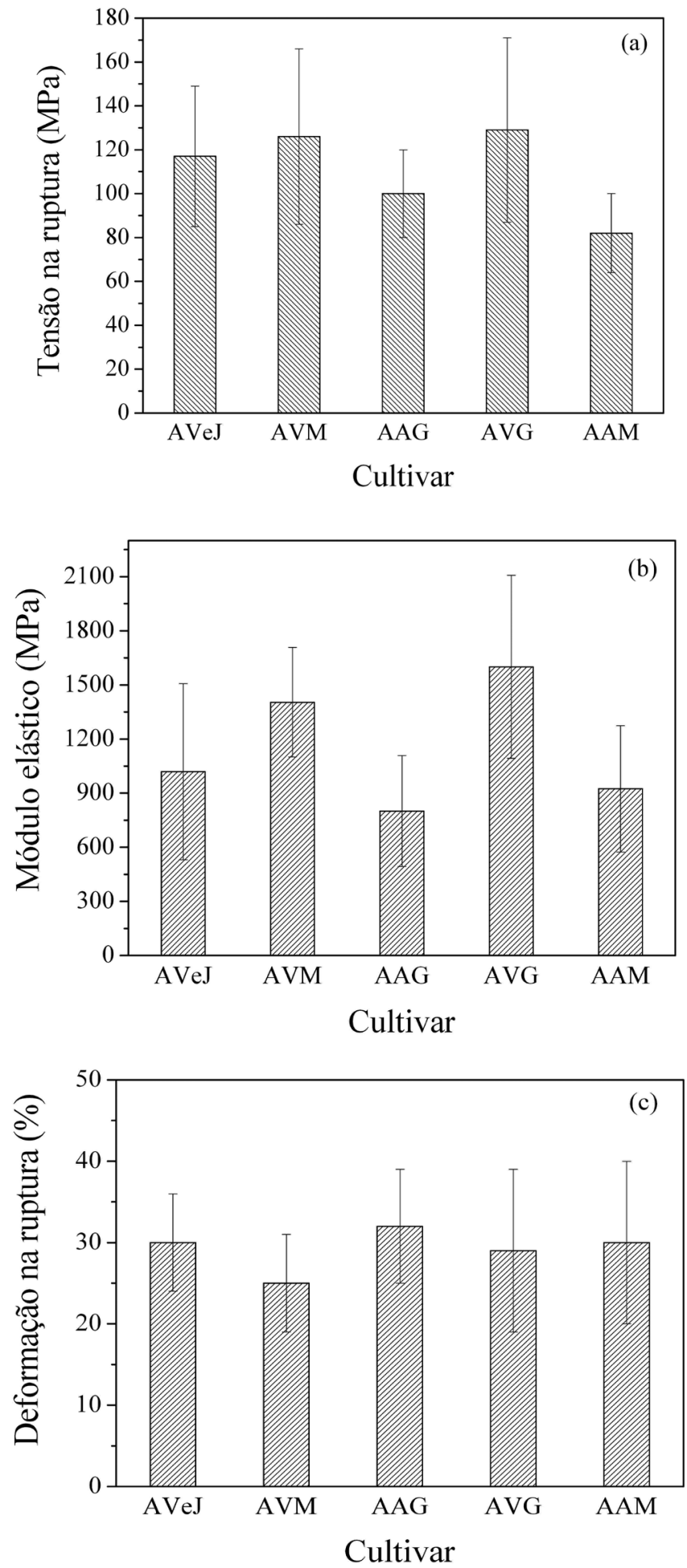

FIGURA 3- Tensão na ruptura (MPa) (a), Módulo de elasticidade (MPa) (b) e deformação na ruptura (\%) (c) para fibra de coco das cultivares: AVeJ, AAM, AVC, AVG e AVM, no ponto de maturação de 210 dias. Sendo AVeJ = Nana 'Anão -Verde'-de-Jequi; AAG = 'Anão-Amarelo'- de-Gramane; AAM = 'Anão-Amarelo'-da-Malásia; AVM = 'Anão-Vermelho'-da-Malásia e AVC = 'AnãoVermelho'-de-Camarões. 

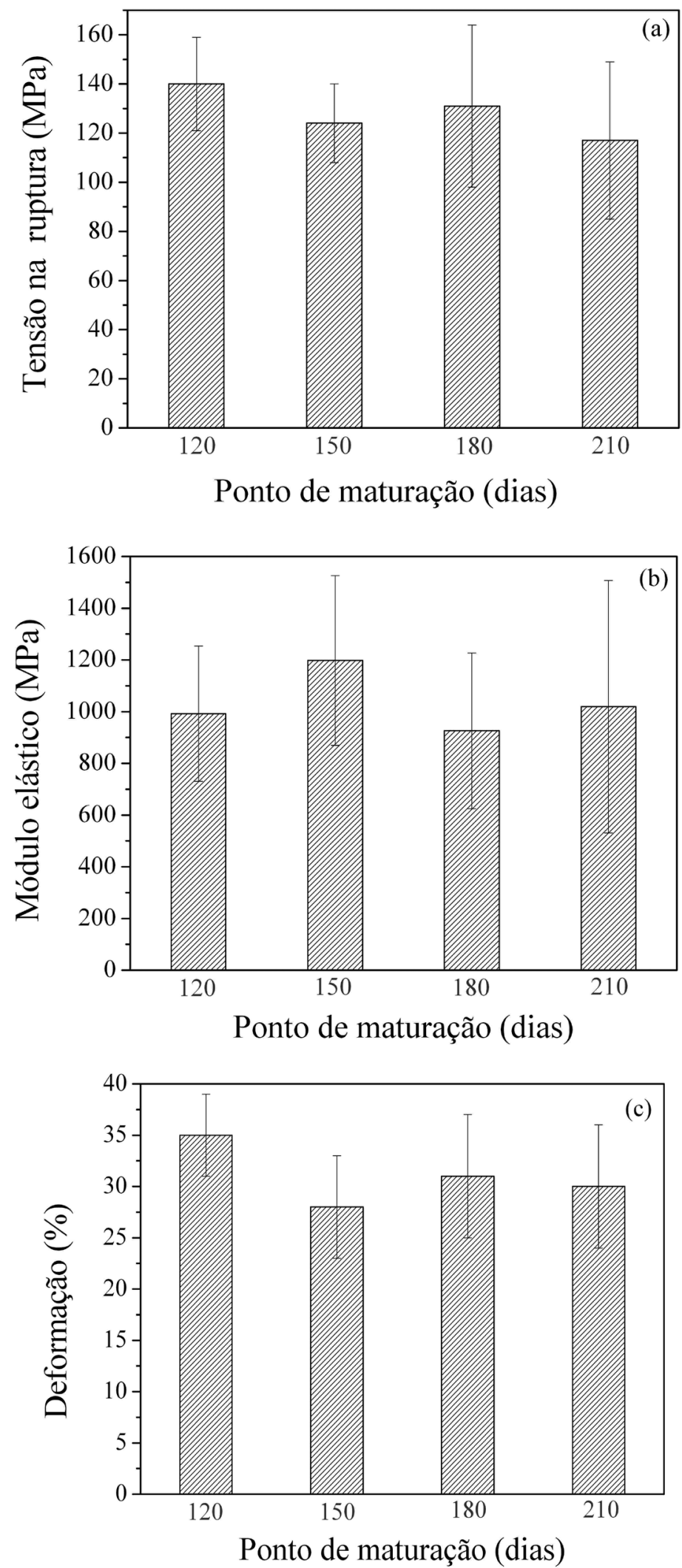

FIGURA 4 - Tensão na ruptura (MPa) (a), Módulo de elasticidade (MPa) (b) e deformação na ruptura (\%) (c) para fibras de coco verde da cultivar Nana 'Anão-Verde'-de-Jequi (AVeJ), em diferentes pontos de maturação. 

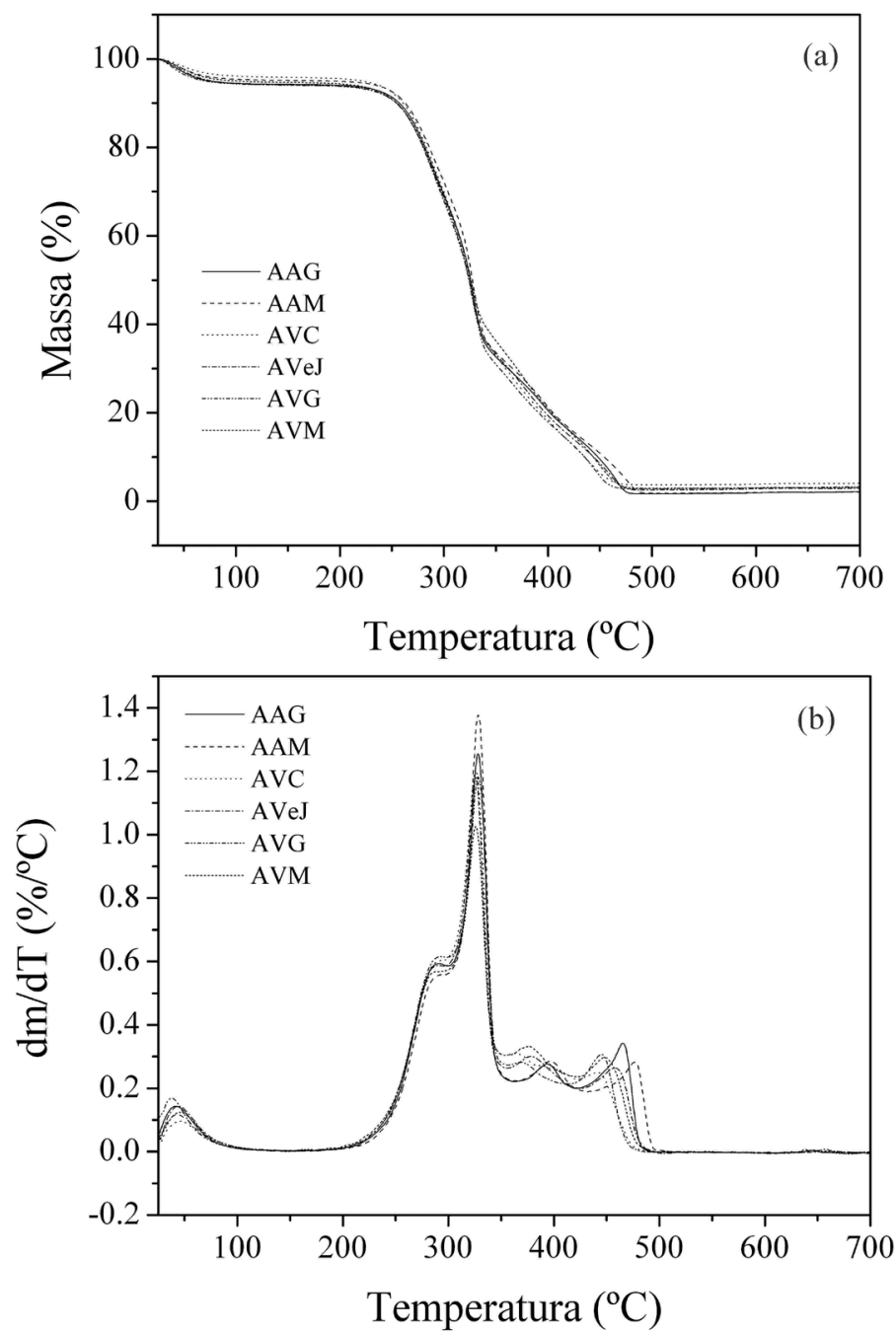

FIGURA 5 - Curvas TG (a) e DTG (b) obtidas para fibras de coco de cultivares AVeJ, AAM, AVC, AVG e AVM com pontos de maturação de 210 dias. Sendo AVeJ = Nana 'Anão-Verde'- de-Jequi; $\mathrm{AAG}=$ 'Anão-Amarelo'-de-Gramane; AAM = 'Anão-Amarelo'-da-Malásia; AVM = 'AnãoVermelho'-da-Malásia e AVC = 'Anão-Vermelho'-de-Camarões.
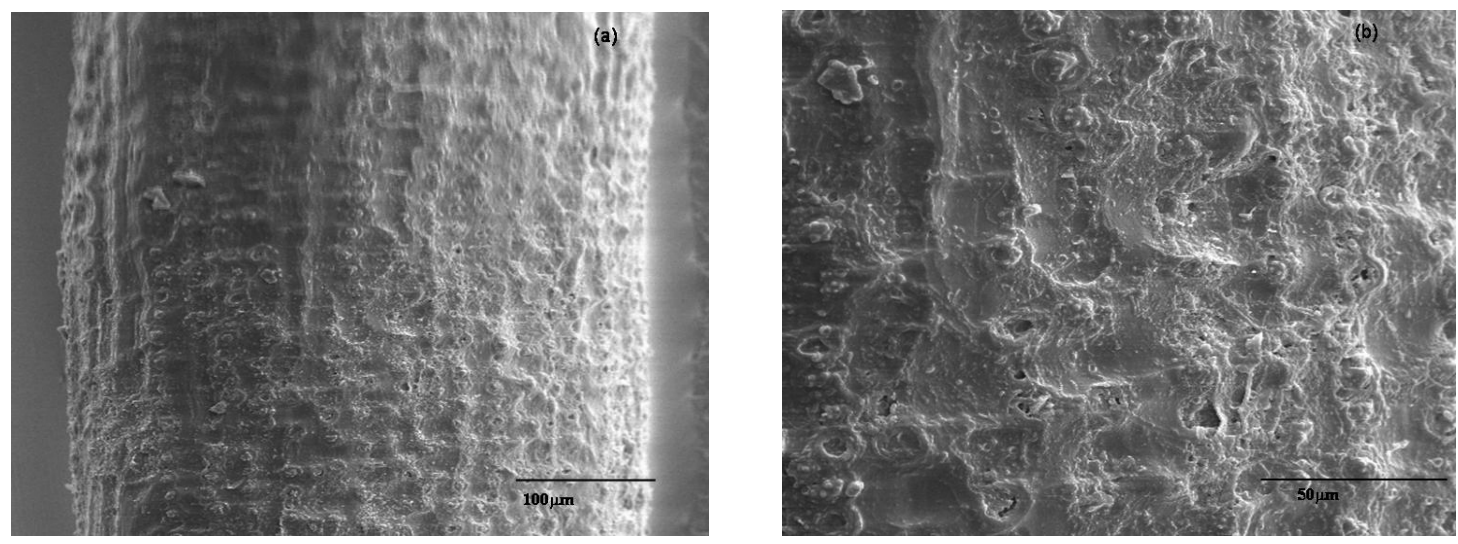

FIGURA 6 - Fotos obtidas por microscopia eletrônica da fibra de coco verde Nana ‘Anão-Verde'-de-Jequi (AVeJ), no ponto de maturação de 150 dias, sendo (a) com aumento de $180 \mathrm{X}$, e (b) com aumento de $500 \mathrm{X}$. 


\section{CONCLUSÃO}

A variação da composição química da fibra de coco verde em função da cultivar foi de $37,2 \pm 0,8 \%$ a $43,9 \pm 0,7 \%$ e de $31,5 \pm 0,1 \%$ a $37,4 \pm 0,5 \%$ para lignina e celulose, respectivamente. A composição química não variou significativamente em função do ponto de maturação para a fibra da cultivar AVeJ. A fibra de coco verde das cultivares e a cultivar AVeJ em diferentes pontos de maturação apresentaram propriedades térmicas e mecânicas similares, as quais são, em geral, próximas às propriedades das fibras de coco maduro. Estes resultados mostram o potencial de utilização da fibra de coco verde de todas as cultivares estudadas como reforços em compósitos.

\section{AGRADECIMENTOS}

Os autores agradecem à FAPESP e ao CNPq, pelo apoio financeiro.

\section{REFERÊNCIAS}

ALVAREZ, V:; VÁZQUEZ, A. Thermal degradation of cellulose derivatives/starch blends and sisal fibre biocomposites. Polymer Degradation and Stability, Essex, v.84, n.1, p.13-21, 2004.

\section{AMERICAN SOCIETY FOR TESTING AND} MATERIALS. ASTM D 3379-89: Standart test method for tensile strenght and Young's modulus for high-modulus single-filament materials. Philadelphia, 1989.

BISMARCK, A.; MOHANTY, A. K.; ARANBERRIASKARGORTA, I.; CZAPLA, S.; MISRA, M.; HINRICHSENBAND, G.; SPRINGERA, J. Surface characterization of natural fibers; surface properties and the water up-take behavior of modified sisal and coir fibers. Green Chemistry, Cambrigde, v.3, n.2, p.100-107, 2001.

BLEDZKI, A. K.; REIHMANE, S.; GASSAN, J. J. Properties and modification methods for vegetable fibers for natural fiber composites. Journal of Applied Polymer Science, New York, v.59, n.2, p.1326-1336, 1996.

BROWNING, B. L. Methods of wood chemistry. New York: Interscience Publishers, 1967. p. 561587.
CARRIJO, O. A.; LIZ, R. S.; MAKISHIMA, N. Fibra de casca de coco verde como substrato agrícola. Horticultura Brasileira, Brasília, v.20, n. 4, p.533$535,2002$.

CORRADINI, E.; GUEDES, C. G. F.; MATTOSO L. H. C.; ROSA D. S. Mechanical, thermal and morphological properties of poly(epsilon-caprolactone)/ zein blends. Polymers for Advanced Technologies, New York, v.15, n.6, p.340-345, 2004.

CORRADINI, E.; DE MEDEIROS, E. S.; CARVAlHO, A. J. F.; CURVELO, A. A. S.; MATTOSO, L. H. C. Mechanical and morphological characterization of starch/zein blends plasticized with glycerol. Journal of Applied Polymer Science, New York, v.101, n.6, p. 4133-4139, 2006.

ECOFLEX $^{\circledR}$ F BX 7011: biodegradable polyester for compostable film: version 1.0. Gabriel Skupin. (S.1.): BASF, 2004. 3 p. Disponível em: $<$ http:// http:// www.basf.com.br/default.asp?id=1216>. Acesso em: 06 jun. 2008.

MOHANTY, A. K.; MISRA, M.; HINRINCHSEN, G. Biofibers, biodegradable polymers and biocomposites: An overview. Macromolecular Materials and Engineering, Berlin, v.276, n.3-4, p.1-24, 2000 .

MOHANTY, A. K.; MISRA, M.; DRZAL, L. T. Novel hybrid coupling agent as adhesion promoter in natural fiber reinforced powder polypropylene composites. Journal of Polymer and Environment, New York, v.10, n.1-2, p.19-26, 2002.

RAZERA, I. A. T. Fibras lignocelulósicas como agente de reforço de compósitos de matriz fenólica e lignofenólica. 2006. 189 f. Tese (Doutorado em Química) - Instituto de Química de São Carlos, Universidade de São Paulo, São Carlos, 2006.

ROSA, M. de F.; ABREU, F. A. P. DE; FURTADO, A. A. L.; BRÍGIDO, A. K. L.; NORÕES, E. R. de V. Processo agroindustrial: obtenção de pó de casca de coco verde. Fortaleza: Embrapa Agroindustria Tropical, 2001. 3p. (Comunicado Técnico, 61).

ROSA, M. de F.; BEZERRA, F. C.; CORREIA, D.; SANTOS, F. J. DE S.; ABREU, F. A. P. de; FURTADO, A. A. L.; BRÍGIDO, A. K. L.; NORÕES, E. R. DE V. Utilização da casca de coco como substrato agrícola. Fortaleza: Embrapa Agroindústria Tropical, 2002. 24 p. (Documentos, 52). 
ROUT, J.; MISRA, M.; TRIPATHY, S. S.; NAYAK, S. K.; MOHANTY, A. K. The influence of fibre treatment on the performance of coir-polyester composites. Composites Science and Technology, Barking, v.61, n.9, p.1303-1310, 2001a.

ROUT, J.; TRIPATHY, S. S.; NAYAK, S. K.; MISRA, M.; MOHANTY, A. K. Scanning electron microscopy study of chemically modified coir fibers. Journal of Applied Polymer Science, New York, v.79, n.7, p.1169-1177, $2001 b$.

ROZMAN, H. D.; TAN, K. W.; KUMAR, R.N.; ABUBAKAR, A.; ISHAK, Z. A. M.; ISMAIL, $H$. The effect of lignin as a compatibilizer on the physical properties of coconut fiber-polypropylene composites. European Polymer, New York, v.36, n.7, p.1483-1494, 2000.

SILVA, G. G.; DE SOUZA, D. A.; MACHADO, J. C.; HOURSTON, D. J. Mechanical and thermal characterization of native Brazilian coir fiber. Journal of Applied Polymer Science, New York, v.76, n.7, p.1197-1206, 2000.

SILVA, R. V.; SPINELLI, D.; BOSE FILHO, W. W.; CLARO NETO, S.; CHIERICE, G. O.; TARPANI, J. R. Fracture toughness of natural fibers/castor oil polyurethane composites. Composites Science Tecnology, Barking, v.66, n.10, p.1328-1335, 2006.

TAPPI TEST METHODS. T 222 om-88: Acid insoluble lignin wood and pulp. Atlanta, 1999.
TOMCZAK, F.; SYDENSTRICKER, T. H. D.; SATYANARAYANA, K. G. Studies on lignocellulosic fibers of Brazil. Part II: Morphology and properties of brazilian coconut fibers. Composites Part AApplied Science and Manufacturing, Kidlinton, v.38, n.7, p.1710-1721, 2007

TRUGILHO, P. F.; LIMA, J. T.; MENDES, L.M. Influência da idade nas características físico-mecânicas e anatômicas da madeira de Eucalyptus saligna. Cerne, Lavras, v.2, n.1, p.97-111, 1996.

VAN DAM, J. E. G.; VAN DEN OEVER, M. J. A.; KEIJSERS, W. E. R. P.; PERALTA, A. G. Process for production of high density/high performance binderless boards from whole coconut husk - Part 1: Lignin as intrinsic thermosetting binder resin. Industrial Crops and Products, Amsterdam, v. 19, n.3, p.207-216, 2004.

YAO, F.; WU, Q.L.; LEI,Y.; GUO, W. H.; XU, Y. J. Thermal decomposition kinetics of natural fibers: Activation energy with dynamic thermogravimetric analysis. Polymer Degradation and Stability, Essex, v.93, n.1, p.90-98, 2008 . 